\title{
Center-Constricted Magnetic Core-Coil Structures for Resonant Wireless Power Transfer
}

\author{
Hirotaka Oshima and Satoshi Shimokawa \\ Fujitsu Laboratories Ltd., 10-1 Morinosato-Wakamiya, Atsugi 243-0197, Japan
}

\begin{abstract}
Magnetic core-coil structures for resonant wireless power transfer are proposed to enhance power transfer efficiency between opposed flat coils. A central region in the direction perpendicular to the plane of a magnetic core plate is constricted with a coil wound closely around the region to increase the figure of merit $k Q$, the product of the coupling coefficient $k$ and the quality factor $Q$. The proposed effect is analyzed in terms of a magnetic circuit model. Electromagnetic field simulation of the systems determines the equivalent circuit parameters and confirms quantitatively the increase in $k Q$ by the center-constricted structures.
\end{abstract}

Key words: wireless power transfer, magnetic core, resonant coil, coupling coefficient, quality factor, magnetic circuit, electromagnetic field simulation

\section{Introduction}

Wireless power transfer using resonant coils ${ }^{1), 2}$ has been attracting considerable attention as a new route to efficient electrical power transfer at a distance without cable. Power transfer efficiency between the coils is determined by the figure of merit $k Q^{3)}$, the product of the coupling coefficient between the coils $(k)$ and the quality factor of the coils $(Q)$.

For higher efficiency, a soft magnetic core with high permeability can be inserted into a coil to increase $k Q$. It is particularly effective for long and narrow solenoidal coils, since the self-inductance $(L)$ and hence $Q$ of such coils are proportional to the permeability of the core ${ }^{4}$. A thin plate core inserted in a flat spiral coil, on the other hand, does not yield such a significant effect due to the demagnetization effect. When the demagnetization factor $\left(N_{\mathrm{d}}\right)$ of a thin plate core is close to unity, $L$ of a flat coil with the plate core can be approximated as $L=L_{0} / N_{\mathrm{d}}$, where $L_{0}$ is the inductance of an air-core coil with the same dimensions ${ }^{4}$. In this case, $L$ is close to $L_{0}$ and independent of the core permeability. Flat spiral coils are often adopted for mobile devices and electric vehicles, and generally used in opposed configurations. Large bulky cores to reduce $N_{\mathrm{d}}$ are, therefore, not practically applicable due to the limited dimensions of the equipment.

Here, we propose center-constricted magnetic core-coil structures that can enhance the efficiency of resonant wireless power transfer without increasing thickness of a plate core inserted in a flat coil. In the following section, the proposed structures are described. The mechanism of the $k Q$ enhancement is explained in terms of a magnetic circuit model in section 3. The effect is confirmed by numerical electromagnetic field simulation by analyzing lumped parameters for an equivalent circuit in section 4. Simulation taking magnetic core loss into account is also performed. The results are discussed in section 5 , and the paper is concluded in section 6 .

\section{Center-constricted magnetic core-coil structures}

\subsection{Power transfer efficiency and $k Q$}

Before describing the proposed magnetic core-coil structures, the relationship between power transfer efficiency $(\eta)$ and $k Q$ in a resonant wireless power transfer system is briefly noted below.

The figure of merit $k Q$ of a two resonant coil system [Fig. 1(a)] can be defined as $k Q=k\left(Q_{1} Q_{2}\right)^{1 / 2}$, where $k$ is the coupling coefficient and $Q_{1}, Q_{2}$ are the quality factors of the resonant coils. By using the self-inductance $L_{1}, L_{2}$, and the mutual-inductance $M_{12}$, $k$ can be written as $k=M_{12} /\left(L_{1} L_{2}\right)^{1 / 2} . Q_{1}, Q_{2}$ of the coils are given by $Q_{1}=\omega L_{1} / R_{1}, Q_{2}=\omega L_{2} / R_{2}$, respectively. Here, $\omega$ is the resonant angular frequency and $R_{1}, R_{2}$ are the electric resistance of the coils. From these equations, $k Q$ of the coils can be written as,

$$
k Q=\omega M_{12}\left(R_{1} R_{2}\right)^{1 / 2} .
$$

When electric power is transferred between the coils, a power source and a load are directly attached to ${ }^{5)}$ or inductively coupled with ${ }^{6), 7)}$ one and the other, respectively. In general, $\eta$ depends on the impedance of the load ${ }^{8}$. When the load impedance is optimally adjusted, the maximum $\eta$ can be described as a function of $k Q$ and given by ${ }^{9)}$,

$$
\eta=(k Q)^{2} /\left[1+\left\{1+(k Q)^{2}\right\}^{1 / 2}\right]^{2} .
$$

In Fig. 1(b), we plot the maximum $\eta$ as a function of $k Q$. It is a monotonically increasing function: the larger $k Q$ gives the larger $\eta$. Consequently, enhancement of $k Q$ is immediately effective in increasing the optimum efficiency of a resonant wireless power transfer system.

\subsection{Magnetic core structures for a flat coil}

We propose here a magnetic core structure for a flat coil to enhance $k Q$ without increasing thickness of a plate core attached to the coil. A schematic example of the structure is shown in Fig. 2(a). A soft magnetic material with high permeability is used for the core. The structure has a constricted region in the center in the direction perpendicular to the plane. A coil is wound 


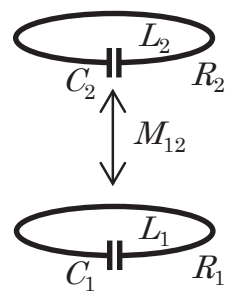

(a) A two resonant coil system

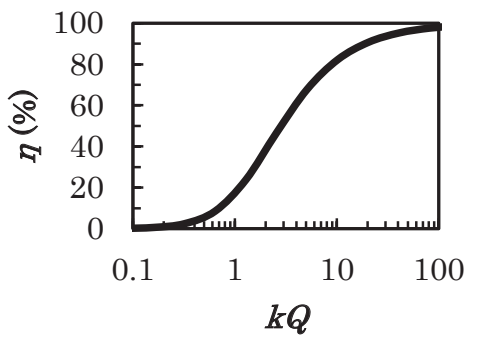

(b) maximum $\eta$ as a function of $k Q$
Fig. 1 (a) A two resonant coil system for resonant wireless power transfer. (b) Maximum $\eta$ as a function of $k Q$.

closely around the constricted region. A capacitor is connected in series with the coil for resonance (not shown in Fig. 2 for simplicity). As shown in Fig. 2(b), the central region with a thickness $y$ has a diameter $D_{\text {c }}$, whereas the outer (upper and lower) regions with a thickness $x$ have a diameter $D_{0}$.

As mentioned in section 1 , a flat coil with a thin plate magnetic core $\left(N_{\mathrm{d}} \sim 1\right)$ inside the coil only shows $k Q$ similar to an air-core coil with the same diameter. Compared to those coils with the diameter $D_{0}$, the proposed magnetic core-coil structure shows larger $k Q$, as will be described below. Note that we use throughout

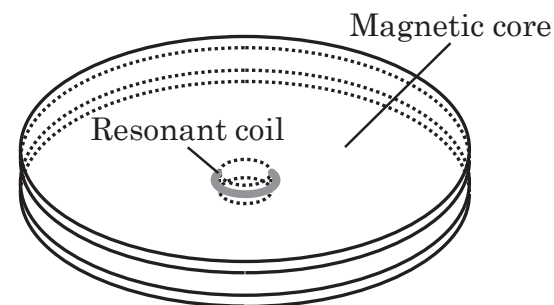

(a) center-constricted magnetic core-coil structure

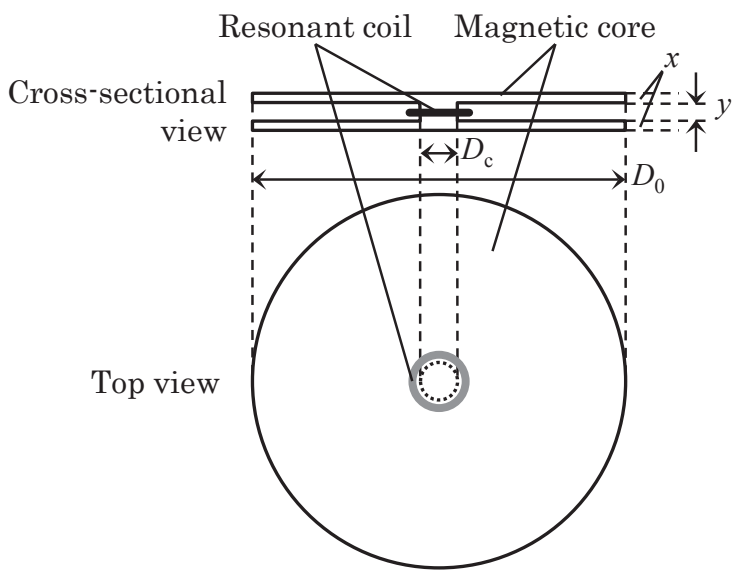

(b) Cross-sectional and top views

Fig. 2 (a) A schematic of a center-constricted magnetic core-coil structure, (b) its cross-sectional and top views. A capacitor for resonance is not shown for simplicity. the paper symmetric circular coils and cores to make the following analysis clear and simple, although similar effect could be obtained in other shapes.

\section{Mechanism of $k Q$ enhancement}

\subsection{Effect of the center constriction}

The essential effect of the center constriction described above can be explained briefly as follows. Suppose one of the resonant coils has the proposed center-constricted magnetic core-coil structure with the parameter $B=D_{0} / D_{\text {c }}$ that represents the degree of the center constriction. Equation (1) signifies that $k Q$ is equal to the ratio of $\omega M_{12}$ to $\left(R_{1} R_{2}\right)^{1 / 2}$. As will be shown below, both of the quantities are decreasing functions of $B$. The point is that, when $B$ increases, relative decrease in $R^{1 / 2}$ is much larger than that in $M_{12}$. This leads to a corresponding increase in $k Q$ with increase in $b$. In the next section, we illustrate why the mutual-inductance $M_{12}$ does not decline as much as the coil resistance $R^{1 / 2}$ when increasing the constriction $B$ in terms of a magnetic circuit model.

\subsection{Magnetic circuit model}

Mutual-inductance between coils is proportional to the interlinkage magnetic flux. Let us suppose that one of the coils is and air-core coil with an area $S_{0}$, and that the interlinkage magnetic flux passing through the air-core coil is $\Phi_{0}$. We then consider replacement of the air-core coil with our center-constricted magnetic core-coil structure having the same projected area $S_{0}$ and the constricted core area $S_{\mathrm{C}}=S_{0} / B^{2}$, as shown in Fig. 3(a). Here the inner diameter of the resonant coil is assumed to be the same as that of the constricted core, and the coil wire width is ignored. In such a case, distribution of the magnetic flux can be analyzed with a magnetic circuit model in analogy with electric current in an electric circuit 10),11). In the proposed structure, magnetic resistance (reluctance) of the center region can be modeled as a parallel circuit of the magnetic resistance of the constricted core and the surrounding air, as illustrated in Fig. 3(b). Assuming that all the magnetic flux $\Phi_{0}$ passes through either the constricted core or the air surrounding the core between the upper and lower plate cores, magnetic resistance of the constricted core and the air are given by $R_{\mathrm{C}}=1 / \mu_{\mathrm{c}} S_{\mathrm{C}}$ and $R_{\mathrm{a}}=1 / \mu_{0}\left(S_{0}{ }^{-} S_{\mathrm{C}}\right)$, respectively, where $l$ is the length of the path and $\mu$ c is the magnetic permeability of the core. As the coil of the structure is wound closely around the constricted core, the interlinkage magnetic flux passing through the coil $\left(\Phi_{\mathrm{C}}\right)$ can be described as,

$$
\begin{aligned}
\Phi_{\mathrm{C}} & =\mu_{\mathrm{C}} S_{\mathrm{C}} \Phi_{0} /\left[\mu_{0}\left(S_{0}-S_{\mathrm{C}}\right)+\mu_{\mathrm{C}} S_{\mathrm{C}}\right] \\
& =\mu_{\mathrm{r}} \Phi_{0} /\left(\mu_{\mathrm{r}}+\beta^{2-1}\right),
\end{aligned}
$$

where $\mu_{\mathrm{r}}$ is the relative permeability of the core material $\left(\mu_{\mathrm{c}}=\mu_{\mathrm{r}} \mu_{0}\right)$. Since the mutual-inductance is proportional to the interlinkage magnetic flux, as mentioned above, the mutual-inductance of the proposed structure $\left(M_{\mathrm{C}}\right)$ is given by,

$$
M_{\mathrm{C}}=\mu_{\mathrm{r}} M_{0} /\left(\mu_{\mathrm{r}}+\beta^{2-1}\right) \text {, }
$$

where $M_{0}$ is the mutual-inductance of the air-core coil. For example, when the relative permeability $\mu_{\mathrm{r}}=1000$ 
and the constriction $B=10, M_{\mathrm{C}}$ is estimated to be $0.91 M_{0}$. This means that the mutual-inductance would be more than $90 \%$ even when the area of the coil is reduced to $1 \%$ of the original one.

On the other hand, the electric resistance of the coil $\left(R_{\mathrm{C}}\right)$ is considered to be proportional to the coil length. $R c$ is, therefore, inversely proportional to $B$. Note that the core loss effect is neglected here. Simulation including the core loss will be shown below in section 5 .

From the above results, when one of the air-core coils is replaced with a center-constricted magnetic core-coil structure, $k Q$ of the system can be written as,

$$
\begin{aligned}
k Q & =\omega M_{\mathrm{C}}\left(R_{1} R_{\mathrm{C}}\right)^{1 / 2} \\
& =\mu_{\mathrm{r}} b^{1 / 2} k_{0} Q_{0} /\left(\mu_{\mathrm{r}}+b^{2-1}\right),
\end{aligned}
$$

where $k_{0} Q_{0}$ is the $k Q$ before the replacement. By substituting $\mu_{\mathrm{r}}=1000$ and $B=10$ again in equation (5), we obtain $k Q=2.9 k_{0} Q_{0}$. In that condition, about three times larger $k Q$ is expected by this new structure. The function $f\left(\mu_{\mathrm{r}}, B\right)=\mu_{\mathrm{r}} B^{1 / 2} k_{0} Q_{0} /\left(\mu_{\mathrm{r}}+B^{2-1}\right)$ can thus be defined as the coefficient of enhancement. We plot in Fig. 4 the coefficient of enhancement $f$ as a function of $b$ for various $\mu_{\mathrm{r}}$. When $\mu_{\mathrm{r}}$ is close to unity, the constriction only gives rise to the degradation in $f$. For larger $\mu_{\text {r }}$, on the other hand, the constriction works effectively for the enhancement and the $f$ becomes several times larger than those without the constriction. We also found that there exists the most effective $B\left(\beta_{\max }\right)$ for each large $\mu_{\text {r. }}$.

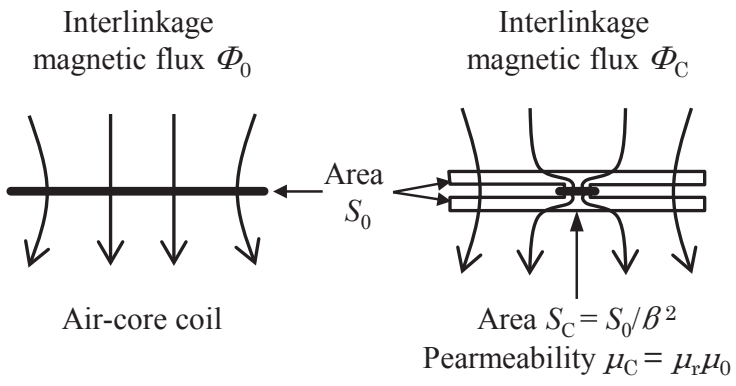

(a) Interlinkage magnetic flux

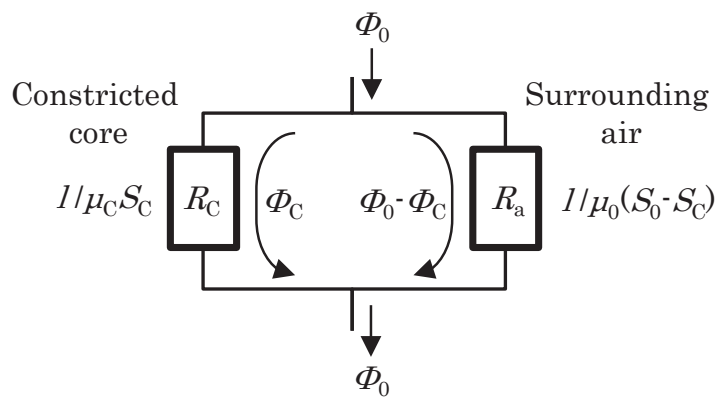

(b) A magnetic circuit model

Fig. 3 (a) Interlinkage magnetic flux through an air-core coil (left) and a center-constricted magnetic core-coil structure with the same projected area (right), (b) A magnetic circuit model for interlinkage magnetic flux through the center-constricted magnetic core-coil structure.

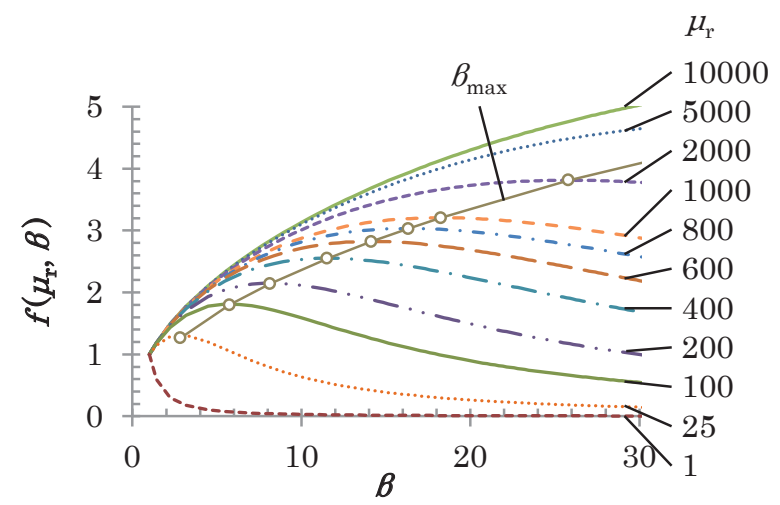

Fig. 4 The coefficient of enhancement $f\left(\mu_{\mathrm{r}}, B\right)$ as a function of $B$ for various $\mu_{\mathrm{r}}$.

\section{Electromagnetic field simulation}

\subsection{Magnetic field distribution}

To confirm the effect of the proposed structure, numerical electromagnetic field simulation has been performed. Lumped parameters (such as inductance and resistance) for an equivalent circuit are directly derived from the field distribution to calculate $k Q{ }^{12}$. Note that nonuniform current distribution in coils due to the skin effect and the proximity effect is explicitly considered in the simulation. We should also note that our previous simulations for various resonant wireless power transfer systems 13-15) have shown good agreement with experiments.

An example of spatial distribution of the magnetic field $H$ and magnetic flux density $B$ is given in Fig. 5 . These figures show a cross section of the system: the amplitude is illustrated by the gradation, and the lines represent a snapshot of magnetic flux. In this example, the upper coil is the proposed center-constricted magnetic core-coil structure, and the lower one is an air-core coil having the same outer diameter for comparison. The outer (upper and lower) core plates have the diameter $D_{0}$ of $100 \mathrm{~mm}$ and the thickness $x$ of $5 \mathrm{~mm}$. The center cnstricted region has the diameter $D_{\mathrm{c}}$ of $D_{0} / 10(B=10)$ and the thickness $y$ of $10 \mathrm{~mm}$. A single-turn coil with the wire width of $1 \mathrm{~mm}$ and thickness of $0.1 \mathrm{~mm}$ is used throughout the paper for both of the air-core and magnetic-core coils to clarify the effect. The inner radius of the magnetic-core coil is set to be $1 \mathrm{~mm}$ larger than that of the center constricted region of the core to avoid the large proximity effect. The frequency is $100 \mathrm{kHz}$ and $\mu_{\mathrm{r}}$ is set to be 1000 .

As described in section 3.2, the concentration of magnetic flux in the center constricted region of the core is necessary for the $k Q$ enhancement. Fig. 5(b) shows that the magnetic flux indeed concentrates in the constriction and mostly passes through the upper coil, which suggests the effectiveness of the proposed center-constricted magnetic core-coil structure. In this calculation, the electric current in the lower air-core coil in Fig. 5 was set to be $1.0 \mathrm{~A}$ as a typical example, and 


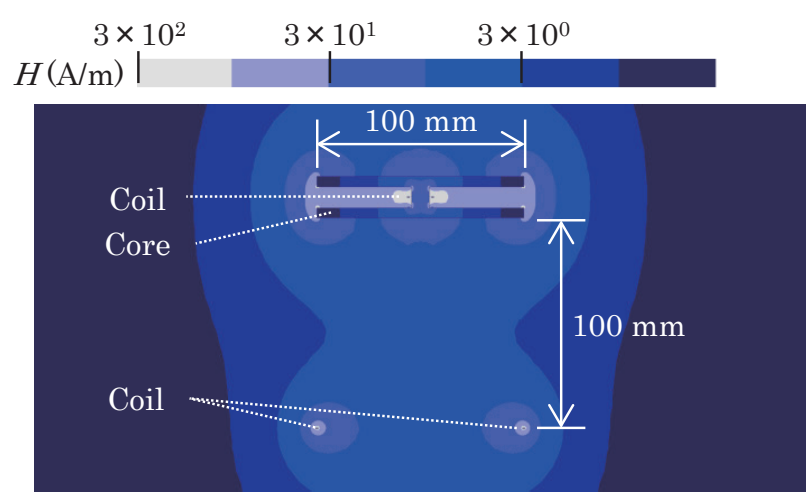

(a) Magnetic field $H$
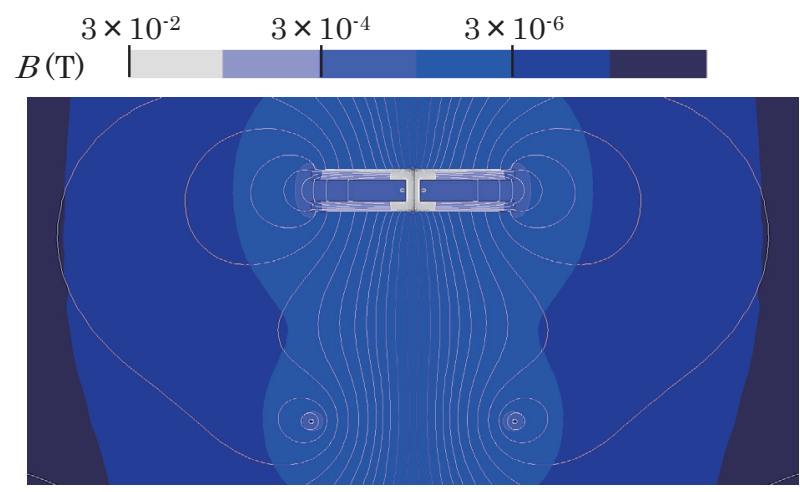

(b) Magnetic flux density $B$

Fig. 5 An example of spatial distribution of (a) magnetic field $H$ and (b) magnetic flux density $B$ around the system. The gradations show log-scale contour plots of $H$ and $B$ divided into six levels. The lines in (b) show a snapshot of magnetic flux.

the current in the upper one in that condition was $2.2 \mathrm{~A}$. It should be noted that the maximum $B$ in the magnetic core in Fig. 5(b) is well below $2 \times 10^{-2} \mathrm{~T}$, which is an order smaller than a typical value of saturation magnetic flux density of ferrite core material.

\subsection{Center-constriction effect on $k Q$}

Fig. 6 presents $k Q$ as a function of $\beta$ for various $\mu_{\mathrm{r}}$, calculated from the lumped circuit parameters deduced from the electromagnetic field simulation. The dimensions of the center-constricted magnetic core-coil structure are identical to those described in section 4.1. An air-core coil with the diameter of $500 \mathrm{~mm}$ is used here for the counterpart to yield a nearly homogeneous field as schematically illustrated in Fig. 3(a). Fig. 6(a) shows the calculated $k Q$ values, whereas Fig. 6(b) shows the $k Q$ normalized by the values at $B=1$ (with no constriction) to clarify the constriction effect. Note that the normalized $k Q$ corresponds to the coefficient of enhancement $f\left(\mu_{\mathrm{r}}, B\right)$ defined in section 3.2.

The results in Fig. 6 clearly show that $k Q$ can be increased by increasing $B$ provided that $\mu_{\mathrm{r}}$ is large enough. When $\mu_{\mathrm{r}}$ is rather small, on the other hand, $k Q$ decreases monotonically. It should also be mentioned that the maximum (normalized) $k Q$ depends on $\mu_{\mathrm{r}}$ and

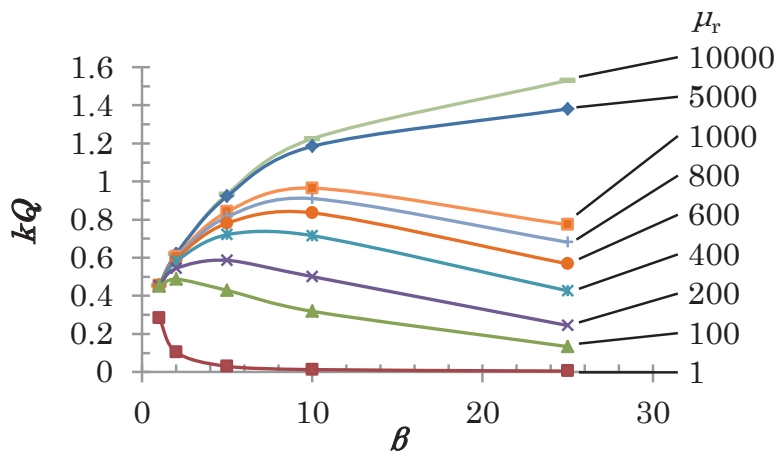

(a) $k Q$

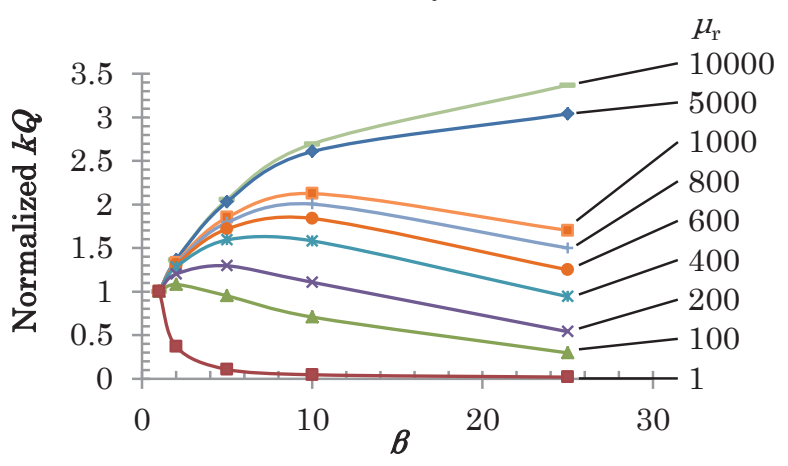

(b) Normalized $k Q$

Fig. 6 (a) Simulated $k Q$ as a function of $B$ for various $\mu_{\mathrm{r}}$. (b) $k Q$ normalized by the values at $B=1$ (with no constriction).

the corresponding $\beta$ increases with the increment of $\mu_{\mathrm{r}}$.

\subsection{Comparison with the magnetic circuit model}

We can find here that the results in Fig. 6(b) described in section 4.2 show $B$-dependent behavior qualitatively similar to those in Fig. 4 derived from the magnetic circuit model. The consistency suggests the validity of the simple model in section 3.2 to understand the basic physics of the $k Q$ enhancement effect.

Further quantitative comparison, however, reveals that the maximum enhancement by the center constriction for each $\mu_{\mathrm{r}}$ calculated by the above simulation is from $60 \%$ to $70 \%$ of the values obtained from the magnetic circuit model. It is probably due to magnetic flux leakage near the angulated constriction and the sharp outer edges of the core. In Fig. 7, we show coil resistance $R_{1}$ of the proposed structure and mutual-inductance $M_{12}$, obtained in the simulation and normalized by the values at $B=1 . R_{1}$ is indeed almost inversely proportional as expected in section 3.1, whereas $M_{12}$ is somewhat smaller than expected from equation (4) for the coefficient of enhancement. The results suggest the existence of magnetic flux leakage larger than predicted by the magnetic circuit model. A closer look at $R_{1}$ also reveals that $R_{1}$ is slightly larger than the $1 / b$ line for large $b$. This is probably due to the small gap between the coil and the core to avoid the proximity effect (as noted in section 4.1), which makes the diameter of the coil slightly larger than the core and 


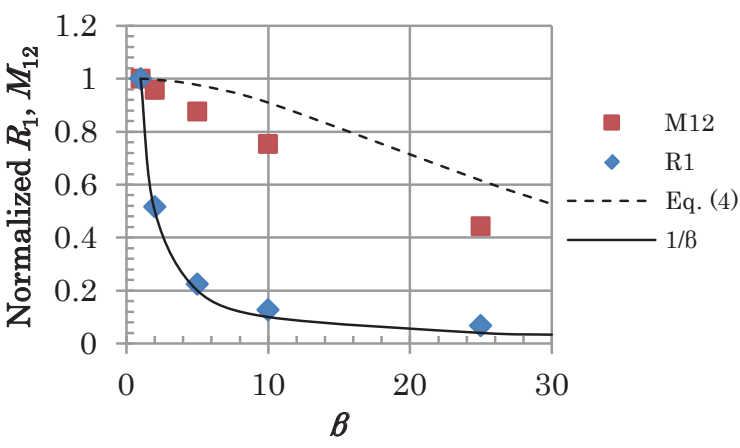

Fig. 7 Normalized $R_{1}$ and $M_{12}$ as a function of $B$ for $\mu_{\mathrm{r}}=1000$. Solid and dashed lines show $1 / B$ and equation (4), respectively.

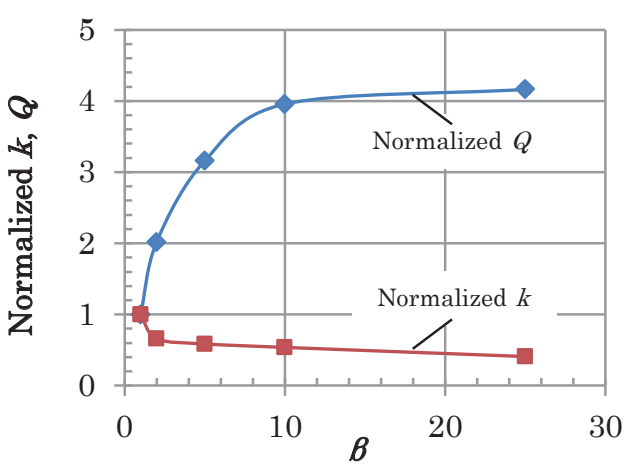

Fig. 8 Normalized $k$ and $Q$ as a function of $B$ for $\mu_{\text {r }}$ $=1000$.

gives rise to increase in $R_{1}$ for large $B$.

It is also important to see the $B$-dependence of $k$ and $Q$ individually for comprehensive understanding of the effect. Fig. 8 shows normalized $k$ and $Q$ as a function of $B$. The results exemplify that, when $B$ increases, $k$ decreases to a certain extent whereas the increase in $Q$ is large enough to outweigh the decrease in $k$. In other words, the center constriction degrades the coupling between the coils to some extent, but it concomitantly amplifies the resonant response of the coil considerably and consequently leads to the enhancement of their product $k Q$.

\subsection{Application to both of the coils}

All the studies shown above have dealt with a combination of a center-constricted magnetic core-coil structure and an air-core coil. It is to clarify the $k Q$ enhancement effect by the proposed structure and better understand the underlying mechanism by comparing them with the magnetic circuit model. It should be added here, however, that the application of the structures to both of the coils (transmitter and receiver) is naturally more effective for the $k Q$ enhancement.

We have confirmed by simulation that the effect by the center constriction is simply multiplied when it is applied to both of the coils. We first calculated $B$-dependence of the normalized $k Q$ between a

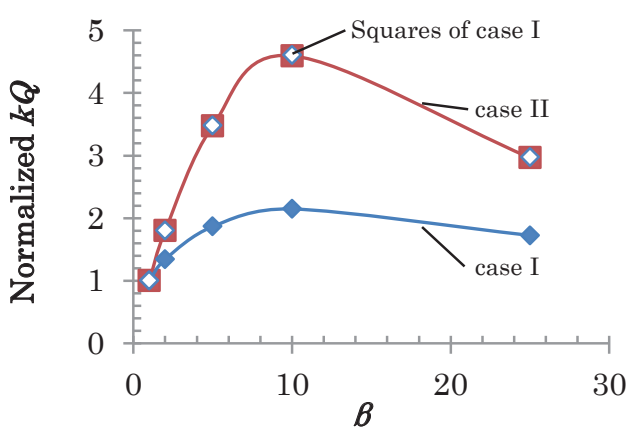

Fig. $9 k Q$ normalized by the values at $B=1$ as a function of $B$ for case I (a center-constricted magnetic core-coil structure and an air-core coil) and case II (two center-constricted magnetic core-coil structures) for $\mu_{\mathrm{r}}=1000$. The squares of the results of case I are also shown for comparison.

center-constricted magnetic core-coil structure and an air-core coil with the same outer diameter (case I). The dimensions of the center-constricted magnetic core-coil structure are identical to those described in sections 4.1 -4.3 and Figs. $6-8$. The pair shown in Fig. 5 is an example of case I. We then calculated the normalized $k Q$ between the two center-constricted magnetic core-coil structures of the same dimensions for various $B$ (case II). We found that the latter is exactly the square of the former for all the same 8 . The results for $\mu_{\mathrm{r}}=$ 1000 are shown in Fig. 9.

The results can be simply explained as follows. As elucidated in section 4.3 , the center constriction of the magnetic core-coil structure leads to some decrease in $k$ and large increase in $Q$ between the coils (and resultant increase in the product $k Q$ ). The effect is independent of a type of the opposite coil. Consequently, the amplification of the normalized $k Q$ is multiplied when we replace both of the coils to the center-constricted magnetic core-coil structures.

\section{Core loss effect}

Magnetic material has, in general, complex magnetic permeability: the imaginary part of permeability is related to the dissipation of energy. We have ignored it in all the simulations discussed above, partly because it is usually small at used frequencies for conventional magnetic core material such as ferrites, and partly because the ideal case should be examined first to make the effect clear and to compare the results with the magnetic circuit model. The loss tangent $(\tan \delta)$ is an index of the energy loss and defined as the ratio of the imaginary part to the real part of permeability, where $\delta$ is the angle in a complex plane. Technically, it is non-zero even in ferrites. To investigate the core loss effect, therefore, we executed electromagnetic field simulation including $\tan \delta$ of magnetic material 13),15).

In Fig. 10, we show $B$-dependence of $k Q$ normalized by the values at $B=1$ for various $\tan \delta$ for $\mu_{\mathrm{r}}=1000$ and 

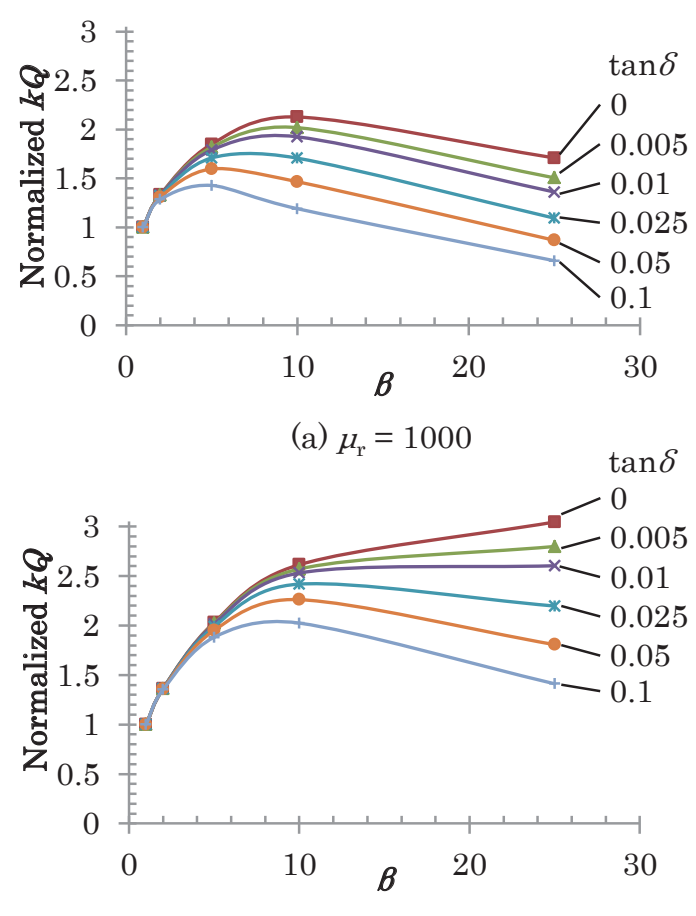

(b) $\mu_{\mathrm{r}}=5000$

Fig. $10 k Q$ normalized by the values at $B=1$ as a function of $B$ for various $\tan \delta$. (a) $\mu_{\mathrm{r}}=1000$, (b) $\mu_{\mathrm{r}}=5000$.

5000. The core-coil system is identical to that employed in section 4.2. Consequently, the results for $\tan \delta=0$ in Fig. 10(a) and 10(b) correspond to the results in Fig. 6(b) for $\mu_{\mathrm{r}}=1000$ and 5000, respectively. As shown in the figures, the increase in $\tan \delta$ in principle leads to the degradation of the $k Q$ enhancement. The energy loss caused by $\tan \delta$ gives rise to increase in the effective electric resistance of the coil and resultant decrease in $Q$. We have confirmed, however, that the $k Q$ enhancement effect is still significant for $\tan \delta \sim 0.01$, which is a typical value for standard ferrite material ${ }^{16)}$.

Additionally, we refer to the core loss factors. In this simulation we represented the core loss by homogeneous $\tan \delta$ due to magnetic hysteresis of core material, which would be appropriate for ferrites. At higher frequencies (typically above $\mathrm{MHz}$ in ferrites), however, the core loss due to displacement currents may also become innegligible, particularly for large cores ${ }^{17)}$. In such cases, the dielectric effect should also be taken into account in simulation.

\section{Conclusion}

We have proposed center-constricted magnetic core-coil structures for opposed flat coils for resonant wireless power transfer. We derived the figure of merit $k Q$ of the coil systems from electromagnetic field simulation by calculating lumped circuit parameters. We have demonstrated that the application of the proposed structures can enhance $k Q$ without increasing the thickness of magnetic cores. The underlying mechanism of the effect can be understood by considering the corresponding magnetic circuit model. The quantitative difference between the results of the simulation and the model analysis is probably due to the simplistic design of the proposed core structures that gives rise to some undesirable leakage of magnetic flux from the wound coils. It suggests that there still is much room for improvement. We have also found that the enhancement effect is basically similar for multi-turn coils. However, the proximity effect, the skin effect, and the dimensions permitted for the coils make the determination of optimal designs more complicated. Further investigation is, therefore, needed to develop the most effective core-coil structures. We believe that the proposed structures are particularly effective for mobile devices as well as electric vehicles, which require efficient power supply but have little space for them. Studies from the perspective of magnetics would help improve the performance of such systems.

\section{References}

1) A. Kurs, A. Karalis, R. Moffatt, J. D. Joannopoulos, P. Fisher, and M. Soljačić: Science, 317, 83 (2007).

2) T. Takura, Y. Ota, K. Kato, F. Sato, H. Matsuki, T. Sato, and T. Nonaka: J. Magn. Soc. Jpn., 35, 132 (2011).

3) A. Karalis, J. D. Joannopoulos, and M. Soljačić: Ann. Phys., 323, 34 (2008).

4) K. Shirakawa, H. Kurata, J. Toriu, H. Matsuki, and K. Murakami: IEEE Trans. Magn., 27, 5432 (1991).

5) Z. N. Low, R. A. Chinga, R. Tseng, and J. Lin: IEEE Trans. Ind. Electron., 56, 1801 (2009).

6) B. L. Cannon, J. F. Hoburg, D. D. Stancil, and S. C. Goldstein: IEEE Trans. Power Electron., 24, 1819 (2009).

7) A. P. Sample, D. A. Meyer, and J. R. Smith: IEEE Trans. Ind. Electron., 58, 544 (2011).

8) I. Awai: IEICE Electron. Express, 10, 20132008 (2013).

9) M. Zargham and P. G. Gulak: IEEE Trans. Biomed. Circuits Syst., 6, 228 (2012).

10) C. J. Carpenter: Proc. IEE, 115, 1503 (1968).

11) S. Chikazumi: Physics of Ferromagnetism, p.17 (Oxford Univ. Press, New York, 1997).

12) S. Shimokawa, H. Kawano, K. Matsui, A. Uchida, and M. Taguchi: 2011 IEEE MTT-S International Microwave Workshop Series on Innovative Wireless Power Transmission: Technologies, Systems, and Applications (IMWS), 219 (2011).

13) H. Kawano, S. Shimokawa, A. Uchida, K. Matsui, K. Ozaki, and M. Taguchi: IEICE Technical Report, WPT2011-15 (2011) (in Japanese).

14) A. Uchida, S. Shimokawa, H. Kawano, K. Ozaki, K. Matsui, and M. Taguchi: IET Microwaves, Antennas and Propagation, 8, 498 (2014).

15) A. Uchida, S. Shimokawa, K. Matsui, H. Kawano, K. Ozaki, and H. Oshima: IEICE Technical Report, WPT2014-42 (2014) (in Japanese).

16) See, for example, FDK standard power material $6 \mathrm{H}$ series: http://www.fdk.com/cyber-e/pdf/FP-FPE001.pdf (As of Jan. 04, 2016).

17) H. Saotome and Y. Sakaki: IEEE Trans. Magn. 33. 728 (1997).

Received Jan. 07, 2016; Accepted Feb. 15, 2016 